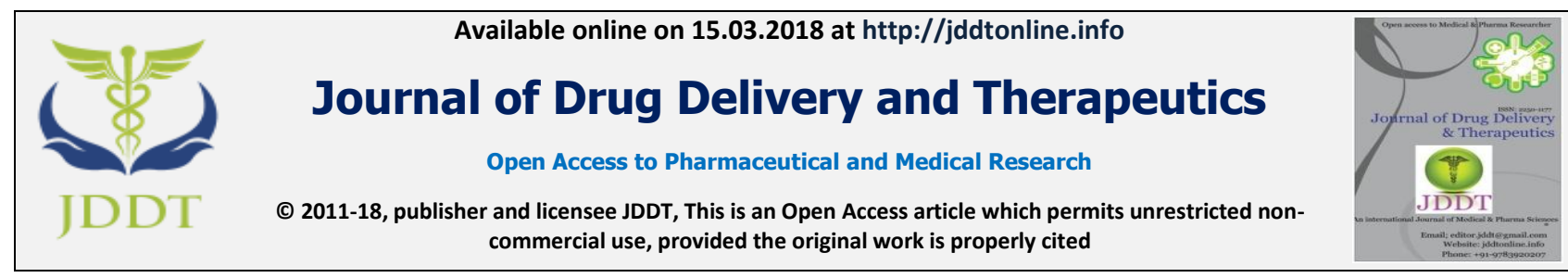

Open 2 Access

Review Article

\title{
TECHNICAL CONSIDERATIONS OF FORCED DEGRADATION STUDIES OF NEW DRUG SUBSTANCES AND PRODUCT: REGULATORY PERSPECTIVES
}

\author{
Singh Asheesh*¹, Singh Parul ${ }^{2}$, Shukla Dilip ${ }^{3}$

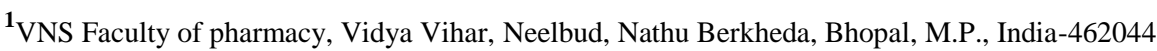 \\ ${ }^{2}$ Department of pharmacy, Guru Ghasidas University, Bilaspur (India)-495009 \\ ${ }^{3}$ Shree Satya Sai University of technical and medical science, sehore M.P, India
}

\begin{abstract}
Forced degradation studies of new drug substances and drug product are important to help develop and demonstrate specificity of stability indicating methods and provide knowledge about possible degradation pathways and degradation products of the active ingredients and help elucidate the structure of the degradants. They were also useful in the investigation of chemical and physical stability of crystal forms. It is better to start degradation studies earlier in the drug development process to have sufficient time to gain more information about the stability of the molecule. This information will in turn help improve the formulation manufacturing process and determine the storage conditions. As no specific set of conditions is applicable to all drug products and drug substances and the regulatory guidance does not specify the conditions to be used, this study requires the experimenter to use common sense. The aim of any strategy used for forced degradation is to produce the desired amount of degradation i.e., 5-20\%. Adequate degradation required to understand the probable degradants for the evaluation of stability indicating method. It has become mandatory to perform stability studies of new drug moiety before filing in registration dossier. The stability studies include long term studies (12 months) and accelerated stability studies (6 months).
\end{abstract}

Keywords: Force Degradation, Stability Indicating, ICH, Drug substances, Drug product.

Article Info: Received 19 Jan, 2018; Review Completed 7 March, 2018; Accepted 8 march, 2018; Available online 15 March, 2018

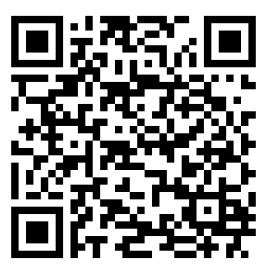

Cite this article as:

Singh A, SinghP, Shukla D, Technical considerations of forced degradation studies of new drug substances and product: regulatory perspectives, Journal of Drug Delivery and Therapeutics. 2018; 8(2):163-168

DOI: http://dx.doi.org/10.22270/jddt.v8i2.1681

*Address for Correspondence

Asheesh Singh, Research Scientist, VNS Faculty of pharmacy, Vidya Vihar, Neelbud, Nathu Berkheda, Bhopal, Madhya Pradesh, India 462044.

\section{INTRODUCTION}

Forced degradation studies are also known as stress testing, stress studies, stress decomposition studies, forced decomposition studies, etc. Forced degradation is a process that involves degradation of drug products and drug substances at conditions more severe than accelerated conditions and thus generates degradation products that can be studied to determine the stability of the molecule. The ICH guideline states that stress testing is intended to identify the likely degradation products which further helps in determination of the intrinsic stability of the molecule and establishing degradation pathways, and to validate the stability indicating procedures used ${ }^{\mathbf{1}, 2}$. But these guidelines are very general in conduct of forced degradation and do not provide details about the practical approach towards stress testing. Although forced Degradation studies area regulatory requirement and scientific necessity during drug development, it is not considered as a requirement for formal stability program. The FDA and ICH guidance's state the requirement of stability testing data to understand how the quality of a drug substance and drug product changes with time under the influence of various environmental factors. Knowledge of the stability of molecule helps in selecting proper formulation and package as well as providing proper 
storage conditions and shelf life, which is essential for regulatory documentation ${ }^{3,4}$.

\section{REGULATORY GUIDELINES}

Various International guidelines recommended forced degradation studies ICH guidelines sometimes apply only to the marketing applications for new products and do not cover the part during clinical development. The $\mathrm{ICH}$ guidelines that are applicable to forced degradation studies are ${ }^{5-7}$

The ICH guidelines that are applicable to forced degradation studies are

ICH Q1A Stability Testing of New Drug Substances and Products

ICH Q1B Photo stability Testing of New Drug Substances and Products

ICH Q2B Validation of Analytical Procedures: Methodology

\section{ICH Q1A (Stress testing):}

Recommended conditions for performing forced degradation studies on drug substances and drug products. The recommendations are to inspect the results of temperature (above that for accelerated testing, i.e., $>50$ C), humidity $(75 \%$ relative humidity $)$, oxidation and photolysis. Wide $\mathrm{pH}$ range should be considered in the testing of solution or suspension. Ultimately the stability-indicating method developed by these samples ${ }^{\mathbf{8}, 9}$.

\section{ICH Q1B:}

Recommended approaches to assessing the photo stability of drug substances and drug products. For drug substance and drug product forced degradation conditions are specified in

Section II and Section III respectively. Forced degradation studies exposure levels are not defined. Solid or in solution/suspension, photo stability testing can be performed. These samples are then used to develop a stability indicating method. Some of the degradation products formed during forced degradation studies may not really be experiential to form during stability studies in which case they need not be examined further ${ }^{10-11}$.

\section{ICH Q2B:}

Gives guidance to validate the analytical methodology. To prove specificity, in section B 1.2.2 (impurities not available) there is a suggestion to utilize samples from forced degradation studies. Whether the analytical method is stability indicating or not 'specificity' is a key factor.

\section{ICH Q3A (R2):}

With respect to both chemistry and safety prospects the identification of each impurity is required. The chemical prospects include classification and identification of impurities, report creation, catalogue of impurities in the specification and a concise discussion of analytical methods. The safety prospects include specific guidance for qualifying those impurities that were not present or were present at significantly minor levels in batch of a new drug substance ${ }^{12-13}$.

\section{Impartial of forced degradation studies}

Forced degradation studies are carried out to achieve the following purposes:

- To establish degradation pathways of drug substances and drug products.

- To differentiate degradation products that is related to drug products from those that are generated from non-drug product in a formulation.

- To determine the intrinsic stability of a drug substance in formulation.

- To reveal the degradation mechanisms such as hydrolysis, oxidation, thermolysis or photolysis of the drug substance and drug product.

- To produce a degradation profile similar to that of what would be observed in a formal stability study under ICH conditions.

- To distinguish degradation products that is related to drug products from those that are generated from non-drug product in a formulation.

- To generate stability indicating nature of a developed method.

- $\quad$ To produce more stable formulations. It also helps in determining the expiry date of a particular formulation.

\section{Time to accomplish forced degradation}

It is very important to know when to perform forced degradation studies for the development of new drug substance and new drug product. FDA guidance states that stress testing should be performed in phase III of regulatory submission process. Stress studies should be done in different $\mathrm{pH}$ solutions, in the presence of oxygen and light, and at elevated temperatures and humidity levels to determine the stability of the drug substance. These stress studies are conducted on a single batch. The results should be summarized and submitted in an annual report. However, starting stress testing early in preclinical phase or phase I of clinical trials is highly encouraged and should be conducted on drug substance to obtain sufficient time for identifying degradation products and structure elucidation as well as optimizing the stress conditions. An early stress study also gives timely recommendations for making improvements in the manufacturing process and proper selection of stability-indicating analytical procedures ${ }^{\mathbf{1 4}-}$ 15

\section{Limits for degradation}

Forced degradation is important part of the drug development process as it provides knowledge about the degradation chemistry of drug substances and drug products. This knowledge is used primarily to develop stability- indicating analytical methods but also useful for other purposes such as formulation development, packaging development and the design of the official stability studies. As there is no formal regulatory guidance for forced degradation, it is recommended to use appropriate conditions to achieve 5-20\% degradation. 


\section{Degradation studies of a drug substance}

FDA demands the following at the time of registration.

> Stressing the drug substance in solution or suspension at alkaline and acidic $\mathrm{pH}$ and under oxidation conditions.

> Stressing the solid bulk drug substance at temperature and temperature + humidity conditions in excess of accelerated conditions.

> Stressing the drug substance photolytically in the solid state or in solution excess.

$>$ Demonstration of the specificity of stability indicating methods with forced degraded samples.

$>$ Full characterization of the degraded products by means of NMR, mass spectrometry (MS), UV analysis
Chemical and physical properties of the degradation products, if available.

$>$ The mechanism and kinetics of degradation products formed, if available ${ }^{\mathbf{1 6}, 17}$.

\section{Strategy for selection of degradation conditions}

Forced degradation is carried out to produce representative samples for developing stabilityindicating methods for drug substances and drug products. The choice of stress conditions should be consistent with the product's decomposition under normal manufacturing, storage, and use conditions which are specific in each case. A general protocol of degradation conditions used for drug substance and drug product is shown in figure no. 1 .

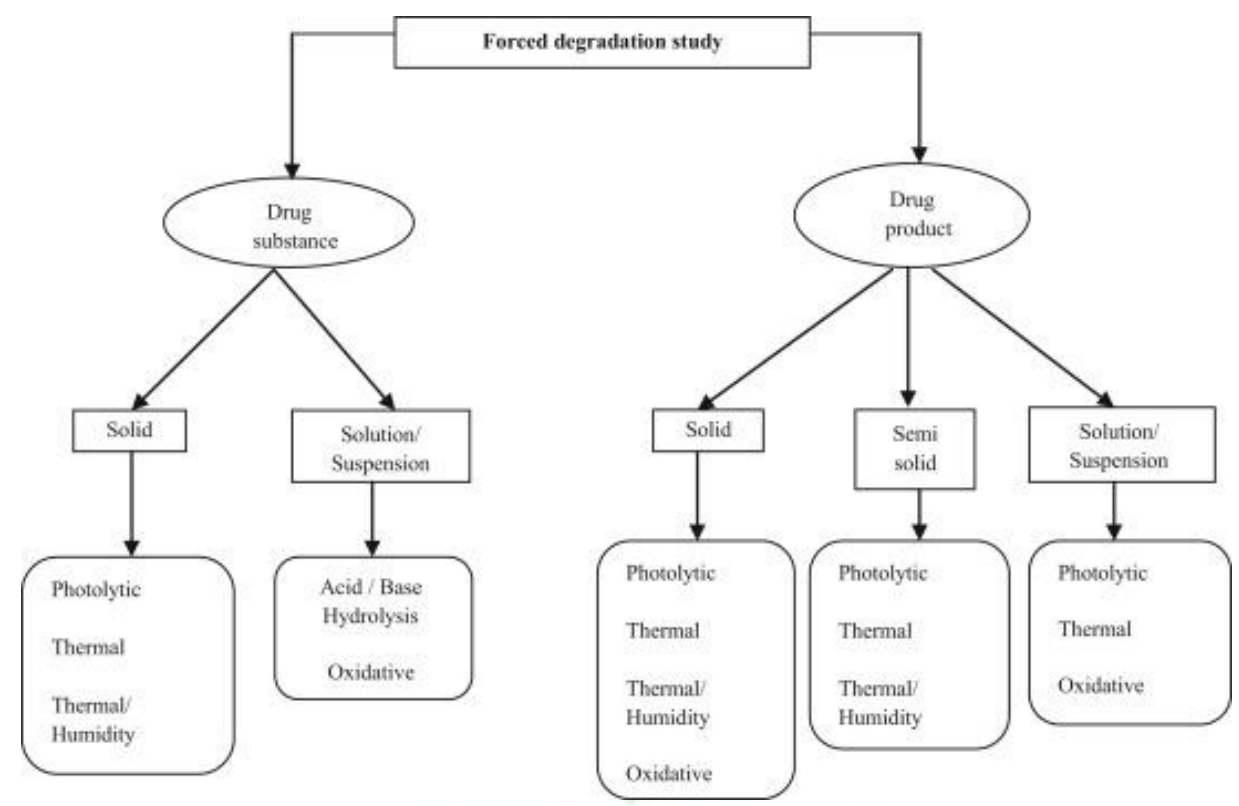

Figure 1: An illustrative flowchart describing various stress conditions used for degradation of drug substance and drug product.

\section{Strategic development of Forced Degradation:}

The structural multiplicity of drug molecules makes it difficult to develop the generic set of conditions for a forced degradation study. The selected stress conditions should be reliable with the product's decomposition ${ }^{\mathbf{1 8}}$, 19. The selected condition should include products property and its degradation under normal manufacturing, storage and use conditions. General protocol Figure 2 illustrates the approach for forced degradation. Forced degradation factors necessary include acid and base hydrolysis, thermal degradation, photolysis, and oxidation and may include freeze-thaw cycles and shear ${ }^{\mathbf{2 0 , 2 1}}$.

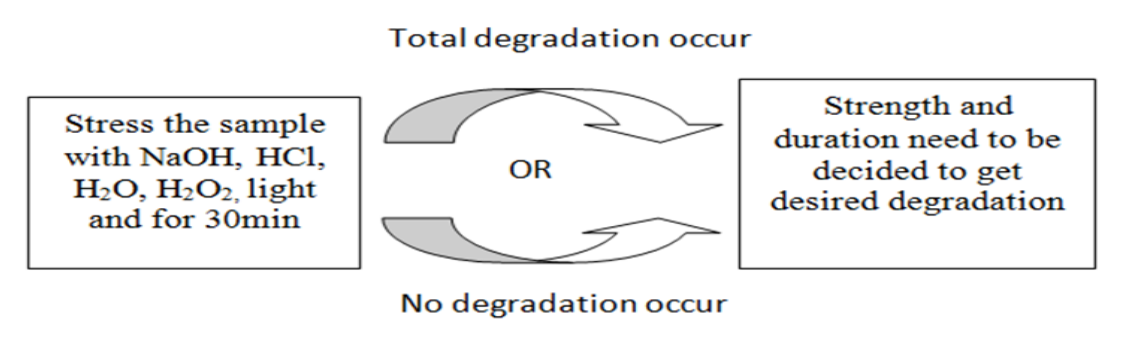

Figure 2: Approach for force degradation

\section{Stability indicating method develop by other analytical technique}

Stability-indicating methods will be characterized by potency, purity and biological activity. The selection of tests is product specific. Stability indicating methods may include various methods like electrophoresis (SDSPAGE, immunoelectrophoresis, Western blot, highresolution chromatography (e.g., reversed phase chromatography, SEC, gel filtration, ion exchange, and 
affinity chromatography) and peptide mapping. The analytical method of choice should be sensitive enough to detect impurities at low levels (i.e., $0.05 \%$ of the analyte of interest or lower) and the peak responses should fall within the range of detector's linearity. The analytical method should be capable of capturing all the impurities formed during a formal stability study at or below ICH threshold limits. Degradation product identification and characterization are to be performed based on formal stability results in accordance with ICH requirements. Conventional methods (e.g., column chromatography) or hyphenated techniques (e.g., LCMS, LC-nuclear magnetic resonance (NMR)) can be used in the identification and characterization of the degradation products. Use of these techniques can provide a better insight into the structure of the impurities that could add to the knowledge space of potential structural alerts for genotoxicity and the control of such impurities with tighter limits. It should be noted that structural characterization of degradation products is necessary for those impurities formed during formal shelf-life stability studies and above the qualification threshold limit ${ }^{22,23}$. By suitable LC-MS conditions, the mass numbers of impurities/ degradants get confirm and further identify the mass of major degradants which are found to be forming greater than $1.0 \%$ during stress studies. LCMS help to illustrate the structures of the major degradants. Similar molecular weights compound may exhibit similar UV profiles, in such cases; attempts must be made to modify the chromatographic parameters to achieve necessary separation. To detect and quantitation of all the potential impurities and degradants an optimal wavelength should be used. The developed method should be validated as per the ICH guidelines $\mathbf{2 4 , 2 5}$.

\section{DEGRADATION CONDITIONS}

\section{Hydrolytic conditions}

Over a wide range of $\mathrm{pH}$ most common degradation, chemical reactions are Hydrolysis The decomposition of a chemical compound by reaction with water is called Hydrolysis. In acidic and basic hydrolysis the catalysis of ionisable functional groups present in the molecule occurs. Forced degradation of a drug substance occurs when the drug interacts with acid and base. It produces primary degradants in the desirable range. Depending on the stability of the drug substance the class and concentrations of acid or base taken should be decided. For acid hydrolysis hydrochloric acid or sulphuric acids (0.1-1 M) considered to be most suitable whereas sodium hydroxide or potassium hydroxides $(0.1-1 \mathrm{M})$ for base hydrolysis are suggested. Co-solvents can be used if compounds are poorly soluble in water. Forced degradation started at room temperature and further temperature increased if there is no degradation ${ }^{26,27}$.

\section{Oxidation conditions}

Hydrogen peroxide is widely used for oxidation of drug substances in forced degradation studies but other oxidizing agents such as metal ions, oxygen, and radical initiators (e.g., azobisisobutyronitrile, AIBN) can also be used. Selection of an oxidizing agent, its concentration, and conditions depends on the drug substance. It is reported that subjecting the solutions to $0.1-3 \%$ hydrogen peroxide at neutral $\mathrm{pH}$ and room temperature for seven days or up to a maximum $20 \%$ degradation could potentially generate relevant degradation products. The oxidative degradation of drug substance involves an electron transfer mechanism to form reactive anions and cations. Amines, sulfides and phenols are susceptible to electron transfer oxidation to give $\mathrm{N}$-oxides, hydroxylamine, sulfones and sulfoxide. The functional group with labile hydrogen like benzylic carbon, allylic carbon, and tertiary carbon or $\alpha$-positions with respect to hetro atom is susceptible to oxidation to form hydro peroxides, hydroxide or ketone ${ }^{28-30}$.

\section{Photolytic conditions}

The photo stability testing of drug substances must be evaluated to demonstrate that a light exposure does not result in unacceptable change. Photo stability studies are performed to generate primary degradants of drug substance by exposure to UV or fluorescent conditions. Some recommended conditions for photostability testing are described in ICH guidelines ${ }^{31}$. Samples of drug substance and solid/liquid drug product should be exposed to a minimum of 1.2 million $\mathrm{lx} \mathrm{h}$ and $200 \mathrm{~W} \mathrm{~h} / \mathrm{m}^{2}$ light. The most commonly accepted wavelength of light is in the range of $300-800 \mathrm{~nm}$ to cause the photolytic degradation. The maximum illumination recommended is 6 million lx h. Light stress conditions can induce photo oxidation by free radical mechanism. Functional groups like carbonyls, nitro aromatic, N-oxide, alkenes, aryl chlorides, weak $\mathrm{C}-\mathrm{H}$ and $\mathrm{O}-\mathrm{H}$ bonds, sulfides and polyenes are likely to introduce drug photosensitivity ${ }^{32,33}$.

\section{Thermal conditions}

Thermal degradation (e.g., dry heat and wet heat) should be carried out at more strenuous conditions than recommended ICH Q1A accelerated testing conditions. Samples of solid-state drug substances and drug products should be exposed to dry and wet heat, while liquid drug products should be exposed to dry heat. Studies may be conducted at higher temperatures for a shorter period. Effect of temperature on thermal degradation of a substance is studied through the Arrhenius equation:

$$
\mathrm{K}=\mathrm{Ae}-\mathrm{Ea} / \mathrm{RT}
$$

where $k$ is specific reaction rate, $A$ is frequency factor, $E a$ is energy of activation, $R$ is gas constant (1.987 cal/deg mole) and $T$ is absolute temperature. Thermal degradation study is carried out at $40-80{ }^{\circ} \mathrm{C}$.

\section{Humidity conditions}

Humidity is one of the effective factors in establishing the potential degradants in the finished product and active pharmaceutical ingredient. Normally 90\% humidity for the duration of one week shall be recommended for the establishment of forced degradation samples ${ }^{34}$.

\section{Stability Indicating Method}

A stability indicating method (SIM) is an analytical procedure used to quantitate the decrease in the amount of the active pharmaceutical ingredient (API) in drug 
product due to degradation. According to an FDA guidance document, a stability-indicating method is a validated quantitative analytical procedure that can be used to detect how the stability of the drug substances and drug products changes with time ${ }^{35}$. A stabilityindicating method accurately measures the changes in active ingredients concentration without interference from other degradation products, impurities and excipients. Stress testing is carried out to demonstrate specificity of the developed method to measure the changes in concentration of drug substance when little information is available about potential degradation product. The development of a suitable stability indicating method provides a background for the preformulation studies, stability studies and the development of proper storage requirements. The RPHPLC is a most widely used analytical tool for separation and quantifying the impurities and it is most frequently coupled with a UV detector. The following are the steps involved for development of SIM on HPLC which meets the regulatory requirements ${ }^{36,37}$.

\section{Sample generation}

For generating samples for SIM the API is force degraded at conditions more severe than accelerated degradation conditions. It involves degradation of drug at hydrolytic, oxidative, photolytic and thermal conditions as discussed earlier. The forced degradation of API in solid state and solution form is carried out with an aim to generate degradation products which are likely to be formed in realistic storage conditions. This sample is then used to develop an SIM ${ }^{38}$.

\section{Method development and optimization}

Before starting the method development, various physiochemical properties like $\mathrm{pKa}$ value, $\log P$, solubility and absorption maximum of the drug must be known, for it lays a foundation for HPLC method development. $\log P$ and solubility helps select mobile phase and sample solvent while pKa value helps determine the $\mathrm{pH}$ of the mobile phase. Reverse phase column is a preferred choice to start the separation of sample components as the degradation is carried out in aqueous solution. Methanol, water and acetonitrile can be used as mobile phase in various ratios for the initial stages of separation. Selection between methanol and acetonitrile for organic phase is based on the solubility of the analyte. Initially the water: organic phase ratio can be kept at 50:50 and suitable modifications can be made as trials proceed to obtain a good separation of peaks. Latter buffer can be added if it is required to obtain better peak separation and peak symmetry. If the method is to be extended to liquid chromatographymass spectrometry (LC-MS), then mobile phase buffer should be MS compatible like triflouroacetic acid and ammonium formate. Variation in column temperature affects the selectivity of the method as analytes respond differently to temperature changes. A temperature in the range of $30-40{ }^{\circ} \mathrm{C}$ is suitable to obtain good reproducibility. It is better to push the drug peak further in chromatogram as it results in separation of all degradation products. Also a sufficient run time after the drug peak is to be allowed to obtain the degradants peak eluting after the drug peak ${ }^{39-41}$.

\section{SUMMARY AND CONCLUSION}

Forced degradation studies are indispensable in the development of stability-indicating and degradant monitoring methods as part of a validation protocol. Forced degradation studies also provide invaluable insight in investigating degradation products and pathways of drug substances and products. Even though the ICH and FDA guidance documents only call for the inclusion of these studies in Phase III of the regulatory submission process, it is strongly recommended these studies be started as early as possible to be able to provide valuable information that can be used to assess the inherent stability of a drug, and to improve formulations and the manufacturing process. Given that no specific set of conditions will be applicable to all drug substances and products, the pharmaceutical scientist should ensure the stress conditions are consistent with product decomposition under normal manufacturing, storage, and intended use conditions. Recommended stress factors include high and low $\mathrm{pH}$, elevated temperature, photolysis, and oxidation. Care should be taken to avoid under-stressing or unduly overstressing the drug substance or product, for this may lead to aberrant and non-representative results. A degradation level of approximately $10 \%$ of the drug substance should be optimal for method optimization.

\section{Acknowledgement}

Authors are thankful to VNS Institute of pharmacy, Neelbad Bhopal to provide necessary facilities to complete the review paper. Especial thanks to Guru Ghasidas Vishwavidyala for providing the necessary facilities and guidance to carry out this review. 


\section{REFERENCES}

1. ICH guidelines, Q1A (R2): Stability Testing of New Drug Substances and Products (revision2), International Conference on Harmonization. 1996.

2. Reynolds DW, Facchine KL, Mullaney JF. Available guidance and best practices for conducting forced degradation studies. Pharmaceutical Technology, 2008; 26:48-56.

3. Singh R, Rehman Z. Current trends in forced degradation study for pharmaceutical product development. Journal of pharmaceutical and educational research, 2012; 2:54-63.

4. Maheswaran R. FDA Perspectives: Scientific Considerations of Forced Degradation Studies in ANDA Submissions. Pharmaceutical Technology, 2012; 36:73-80.

5. Singh S, Bakshi M. Guidance on conduct of stress tests to determine inherent stability of drugs. Phrama Technology, 2000, 24, 1-14.

6. Boccardi G. Oxidative susceptibility testing, pharmaceutical Stress Testing-Predicting Drug Degradation; Baertschi SW, editors, Taylor and Francis, New York. 2005.

7. Alsante KM, Hatajik TD, Lohr LL, Santafianos D Sharp TR. Solving impurity/degradation problems: case studies. In; handbook of Isolation and Characterization of impurities in Pharmaceutical, Ahuja S, Alsante K, editors, Academics Press, New York. 2003, 380-383.

8. Alan RO, Brigitte ES, Yanqiu SA, Polshyna NM, Dunphy R, Barbara LM. Forced degradation studies of rapamycin: Identification of autoxidation products. Journal Pharmaceutical and Biomedical analysis, 2012; 59:194-200.

9. Trabelsi H, Hassen IE, Bouabdallah S, Bouzouita K, Safta F. Stability indicating LC method for determination of Pipamperone. Journal of Pharmaceutical and Biomedical Analysis, 2005; 39:914-919.

10. Bojana P, Markus D, Kappe CO. Microwave-assisted forced degradation using high-throughput microtiter platforms. Journal of Pharmaceutical and Biomedical Analysis, 2011; 56:867-873.

11. Baertschi SW, Thatcher SR. Sample presentation for photostability studies: problems and solutions, In; Pharmaceutical Photostability and Stabilization Technology. Piechocki J, Editor, Taylor \& Francis, New York. 2006; 445450.

12. Baertschi SW, Alsante KM. Stress testing: the chemistry of the drug degradation, In; Pharmaceutical Stress Testing, Baertschi SW, editors, Taylor \& Francis, New York. 2005; 99-101.

13. FDA Guidance for Industry, INDs for Phase 2 and 3 Studies of Drugs, Including Specified Therapeutic Biotechnology Derived Products, Draft Guidance, Food and Drug Administration.

14. Jenke DR. Chromatographic method validation: a review of common practices and procedures II. Journal of Liquid Chromatography, 1996; 19:737-757.

15. Ali NW, Abbas AS, Zaazaa HE. Validated stability indicating methods for determination of Nitazoxanide in presence of its degradation products, Journal Pharmaceutical Analysis. 2012; 2:105-116

16. Carstensen JT. Solution Kinetics; Kinetic pH profiles; Oxidation in solution; Catalysis, Complexation, and Photolysis, in Carstensen JT, Rhodes CT, Drug Stability, Principle and Practice, 3rd edition, Marcel Dekker, New York, 2000, 19-143.

17. David A, Alexander TF. Fast track: Physical Pharmacy, 1st edition, London, Pharmaceutical Press, 2008, 29-31.

18. ICH, Final Guidance on Stability Testing of Biotechnological/Biological Products Availability, International Conference on Harmonization, 1996.

19. ICH Guidance for Industry, Q1B: Photo stability testing of New Drug Substances and Product, International Conference on Harmonization, 1996.
20. ICH Guidance for Industry, Q2B: Validation of Analytical Procedures: Methodology, International Conference on Harmonization, 1996.

21. Ngwa G. Forced degradation studies as an integral part of HPLC stability indicating method development. Drug Delivery Technology, 2010; 10:56-59.

22. Sitaram C, Rupakula R, Reddy BN. Determination and characterization of degradation products of Anastrozole by LC MS/ MS and NMR spectroscopy. Journal of Pharmaceutical and Biomedical Analysis, 2011; 56:962-968.

23. Skoog DA. West DM Principles of Instrumental Analysis. Saunderg Golden, Japan, 1980, 2-3.

24. Dolan J. Stability-indicating assays: LC trouble shooting. LCGC, 2002; 20:346-349.

25. Smela JW. Regulatory considerations for stability indicating analytical methods in drug substance and drug product testing. Pharmaceutical Review, 2002; 8:51-54.

26. Maheswaran R. FDA perspectives: scientific considerations of forced degradation studies in ANDA submissions. Pharmaceutical Technology, 2012; 36:73-80.

27. Kovarikova P, Jiri K, Jiri D, Lucie T. HPLC study of glimepiride under hydrolytic stress conditions. Journal of Pharmaceutical and Biomedical Analysis, 2004; 36:205-209.

28. FDA Guidance for Industry, INDs for Phase II and III StudiesChemistry, Manufacturing, and Controls Information. Food and Drug Administration, 2003, 1-27.

29. Singh S, Bakshi M. Guidance on conduct of stress tests to determine inherent stability of drugs. Pharmaceutical Technology, 2000, 24, 1-14.

30. Riddhiben MP, Piyushbhai MP, Natubhai MP. Stability indicating HPLC method development-a review. International Research Journal of Pharmacy, 2011; 2:79-87.

31. ICH Guidance for Industry, Q1B: Photo stability Testing of New Drug Substances and Product, International Conference on Harmonization.1996.

32. Baertschi SW, Thatcher SR. Sample presentation for photo stability studies: problems and solutions, in: J. Piechocki (Ed.), Pharmaceutical Photostability and Stabilization Technology, Taylor \& Francis, New York, 2006, 445-450.

33. Allwood M, Plane J. The wavelength-dependent degradation of vitamin A exposed to ultraviolet radiation, International Journal of Pharmacy 1986; 31:1-7.

34. ICH Q3A (R2) Impurities in New Drug Substances and Products (Step 4), International Conference on Harmonization, 2006, 1-15.

35. Phale MD, Hamrapurkar PD. Optimization and establishment of a validated stability-indicating HPLC method for study of the stress degradation behavior of metoprolol succinate. Journal of AOAC International. 2010, 93, 911-916.

36. Wartz M, Krull I. Developing and Validating StabilityIndicating Methods. LCGC, 2005, 26, 23586-23593

37. Ngwa G. Forced Degradation Studies. Drug Delivery Technology, 2010, 10, 3, 0976-1225.

38. Reynolds DW. Forced Degradation of Pharmaceuticals. Pharmaceutical Review, 2004, 23, 56-61.

39. European Medicines Agency, Guidance for Industry Q2B Validation of Analytical Procedures: Methodology. 2011.

40. Alsante KM, Ando A, Brown R, Ensing J, Hatajik TD, Tsuda, Y. The Role of Degradant Profiling in Active Pharmaceutical Ingredients and Drug Products. Advance Drug Delivery Review, 2007, 1, 59-65

41. Klick S, Muijselaar PG, Waterval J, Eichinger T, Korn C, Gerding TK, Debets AJ, Sanger-Van de Griend C, Van den Beld, C, Somsen GW, De Jong GJ. Toward a Generic Approach for Stress Testing of Drug Substances and Drug Products. Pharmaceutical Technology, 2005, 29, 48-57. 